Mathematical Modelling and Analysis

Volume 6 Number 2, 2001, pages 241-250

(C) 2001 Technika

\title{
ON REPRESENTATION FORMULA FOR SOLUTIONS OF HAMILTON-JACOBI EQUATION FOR SOME TYPES OF INITIAL CONDITIONS
}

\author{
G. GUDYNAS
}

Klaipèda University, Department of System Research

H. Manto 84, Klaipėda LT-58008

E-mail: ggintaut@gmf.ku.lt

Received June 2, 2001; revised September 20, 2001

\begin{abstract}
This article investigates the representation formula for the semiconcave solutions of the Cauchy problem for Hamilton-Jacobi equation with the convex Hamiltonian and the unbounded lower semicontinous initial function. The formula like Hopf's formula is given by forming envelope of some fundamental solutions of the equation.
\end{abstract}

\section{INTRODUCTION}

In this paper we are concerned with the Cauchy problem

$$
\begin{aligned}
& u_{t}+\left(A(x) u_{x}, u_{x}\right)=0, \\
& u(0, x)=\varphi(x)
\end{aligned}
$$

in $S_{T}=\left\{t \in(0, T), x \in R^{n}\right\}$. We investigate the generalized solutions of (1.1), (1.2), because the classical solutions exist in general only in some neighborhood of hyperplane $\{t=0\}$. There are two ways to define the generalized solution. The one of them, when the Hamiltonian is convex, is to investigate the semiconcave solutions [4], [5], [9]. The other way is to work with the viscosity solutions of (1.1), (1.2) [2], [3]. The nice property of solutions of differential equations of first order is that the envelope of family of solutions is also solution of this equation. The various representation formulas for solutions of Cauchy problem for Hamilton-Jacobi equations are based on this 
property [1], [5], [7], [10]. For the equation

$$
u_{t}+H\left(u_{x}\right)=0,
$$

when $H$ convex, and $\varphi$ is Lipschitz continuous in $R^{n}$, E. Hopf [7] introduced the formula for solutions of (1.3), (1.2)

$$
u(t, x)=\min _{\xi \in R^{n}}\left[\varphi(\xi)+t \Phi\left(\frac{x-\xi}{t}\right)\right]
$$

where $\Phi(q)=\sup _{p \in R^{n}}((q p)-H(p))$ is Legrende transform of $H$.

In first section of this work we find a formula like this for the solutions of (1.1), (1.2), when the matrix $A(x) \in C^{2, \alpha}\left(R^{n}\right)$ satisfies

$$
a_{1}|\xi|^{2} \leq(A(x) \xi, \xi) \leq a_{2}|\xi|^{2}, \quad a_{1}, a_{2}>0,
$$

and the initial function $\varphi(x)$ is lower semicontinous and

$$
\lim _{|x| \rightarrow \infty}|x|^{-2}|\varphi(x)|=0 .
$$

In second section we give formulas for the solutions of Cauchy problem for (1.3) and conservation law

$$
u_{t}+H\left(u_{x}\right)=0
$$

when $x \in R$ and the initial functions are respectively piecewise linear and piecewise constant.

\section{THE REPRESENTATION FORMULA FOR SOLUTIONS OF (1.1), (1.2) WITH THE UNBOUNDED INITIAL DATA}

We give some definition of the generalized solution of $(1.1),(1.2)$ in the sense of work [9]. First we suppose that the initial function $\varphi(x)$ is Lipschitz continuous on $R^{n}$.

Definition 2.1. The Lipschitz continuous function $u(t, x)$ in $\bar{S}_{T}=\{t \in[0, T]$,

$\left.x \in R^{n}\right\}$ is called the generalized (semiconcave) solution of (1.1), (1.2) if $u(t, x)$ solves (1.1) a.e. on $S_{T}$, satisfies (1.2), and the inequality

$$
u(t, x+l)-2 u(t, x)+u(t, x-l) \leq C_{n}|l|^{2}
$$

holds for all points $(t, x),(t, x+l),(t, x-l) \in S_{T}^{n}=\left\{t \in\left[\frac{1}{n}, T\right],|x| \leq n\right\}$. 
For the unbounded, lower semi-continuous initial function we suppose such definition of generalized solution of (1.1), (1.2).

Definition 2.2. The locally Lipchitz continuous function on $S_{T}$ is called the generalized (semiconcave) solution of $(1.1),(1.2)$ if $u(t, x)$ solves (1.1) a.e. on $S_{T}$ and satisfies:

$$
\Delta_{l}^{2} u(t, x)=u(t, x+l)-2 u(t, x)+u(t, x-l) \leq C_{n}|l|^{2}
$$

for any $(t, x),(t, x+l),(t, x-l) \in S_{T}^{n}$,

$$
\lim _{|x| \rightarrow \infty}|x|^{-2}|u(t, x)|=0
$$

uniformly with respect $t \in[0, T]$,

$$
\lim _{t \rightarrow 0} u(t, x)=\varphi(x)
$$

Now we will use the results of work [6]. In this work we investigated the boundary problem

$$
\begin{aligned}
& \left(A(x) u_{x}, u_{x}\right)=1, \\
& u \|_{\partial \Omega}=\varphi
\end{aligned}
$$

Definition 2.3. The Lipschitz continuous function $u(x)$ in $\bar{\Omega}$ is called the generalized (semiconcave) solution of $(2.2),(2.3)$ if $u(x)$ solves $(2.2)$ a.e. on $\Omega$, satisfies (2.3) and the inequality

$$
u(x+l)-2 u(x)+u(x-l) \leq C_{\delta}|l|^{2}
$$

holds for all points $x, x+l, x-l \in \Omega_{\delta}$, where $\Omega_{\delta}=\{x \in \Omega: \operatorname{dist}(x, \partial \Omega) \geq$ $\left.\delta,|x| \leq \frac{1}{\delta}\right\}, \delta>0$

In the case, when domain $\Omega$ is unbounded, we additionally require

$$
\lim _{\substack{|x| \rightarrow+\infty \\ x \in \Omega}} u(x)=+\infty .
$$

Consider the generalized solution $u^{r}(x, \xi)$ of $(2.2)$ on $R^{n} \backslash B_{r}(\xi), B_{r}(\xi)=\{$ $\left.x \in R^{n}:|x-\xi|<r\right\}$, satisfying boundary conditions

$$
\left.u^{r}\right|_{S_{r}(\xi)}=0
$$


where $S_{r}(\xi)=\left\{x \in R^{n}:|x-\xi|=r\right\}$. Fix any $\xi \in R^{n}$. Define the function

$$
G(x, \xi)=\left\{\begin{array}{c}
\lim _{r \rightarrow 0} u^{r}(x, \xi), \text { when }|x-\xi|>r_{0}, \forall r_{0}>0, \\
r<r_{0} \\
0, \text { when } x=\xi .
\end{array}\right.
$$

We concluded in this work that the function $G(x, \xi)$ is semiconcave solution of (2.2) on $R^{n} \backslash\{\xi\}$ such that

$$
\frac{1}{\sqrt{a_{2}}}|x-\xi| \leq G(x, \xi) \leq \frac{1}{\sqrt{a_{1}}}|x-\xi|
$$

for every $x, \xi \in R^{n}$ and is continous with respect $\xi$.

Now we prove the theorem.

Theorem 2.1. Let the matrix $A(x)$ satisfies (1.5) and the initial function $\varphi(x)$ is lower semicontinuous on $R^{n}$ and satisfies (1.6). Then there exists the unique semiconcave solution of (1.1), (1.2) on $S_{T}$ which can be represented by formula

$$
u(t, x)=\min _{\xi \in R^{n}}\left\{\varphi(\xi)+\frac{G^{2}(x, \xi)}{4 t}\right\} .
$$

Proof. According to (1.6), (2.6) the minimum exists. Define the point $\xi_{0}$ which gives the minimum of $I(t, x, \xi)=\varphi(\xi)+\frac{G^{2}(x, \xi)}{4 t}$. Fix any point $\xi \in R^{n}$. We can calculate that $I(t, x, \xi)$ satisfies (1.1). Let $(t, x) \in S_{T}$. Then $\frac{I(t, x, \xi)}{|l|^{2}}$ is bounded on $S_{T}^{n}=\left\{t \in\left[\frac{1}{n}, T\right], \frac{1}{n} \leq|x-\xi| \leq n\right\}$ if $\frac{\Delta_{l}^{2} G^{2}(x, \xi)}{|l|^{2}}$ is bounded when $x=\xi,|l| \neq 0$. From (2.6) we deduce

$$
\frac{\Delta_{l}^{2} G^{2}(\xi, \xi)}{|l|^{2}}=\frac{G^{2}(\xi+l, \xi)-2 G^{2}(\xi, \xi)+G^{2}(\xi-l, \xi)}{|l|^{2}} \leq \frac{2}{a_{1}} .
$$

Now suppose that $\varphi(x)$ is bounded on $R^{n}$, and we will prove the theorem in this case. Let $|\varphi(x)| \leq M$, then

$$
u(t, x)=\min _{\xi \in R^{n}}\{I(t, x, \xi)\}=I\left(t, x, \xi_{0}\right) \leq I(t, x, x) \leq \varphi(x) \leq M .
$$

On the other hand, we have

$$
u(t, x) \geq \min _{\xi \in R^{n}}\{\varphi(x)\} \geq-M
$$

Consequently

$$
|u(t, x)| \leq \sup _{\xi \in R^{n}}|\varphi(x)|
$$


on $S_{T}$. According to Lemma 1 , [5] we have that $u(t, x)$ solves $(1.1)$. Now we will prove that $u(t, x)$ satisfies initial condition (1.2). From (2.8) we deduce

$$
\frac{G^{2}\left(x, \xi_{0}\right)}{4 t} \leq 2 M
$$

In view of (2.6), in computing the minimum of $I(t, x, \xi)$ we need only consider the set $O(t, x)=\left\{\xi:|x-\xi|^{2} \leq 8 M a_{2} t\right\}$. According to (2.7), (2.8) we see

$$
\inf _{\xi \in O(t, x)} \varphi(\xi) \leq u(t, x) \leq \varphi(x) .
$$

From this inequality we have $\lim _{t \rightarrow 0} u(t, x)=\varphi(x)$, because the semicontinuity of $\varphi(x)$ implies

$$
\lim _{t \rightarrow 0} \inf _{\xi \in O(t, x)} \varphi(\xi)=\varphi(x) .
$$

Now we prove the uniqueness of solution representing by formula (2.7). First we suppose, that $\varphi(x)$ is Lipschitz on $R^{n}$. We will prove that $u(t, x)$ Lipschitz on $\bar{S}_{T}$, too. Because (2.6) implies that $G(x, \xi)$ is bounded on $O(t, x)$, then according to (1.5), (2.2) we see

$$
\left|G_{x}(x, \xi)\right| \leq \frac{1}{\sqrt{a_{1}}}
$$

a.e. on $S_{T, n}=\left\{t \in\left[\frac{1}{n}, T\right], x \in R^{n}\right\}$, thus $u(t, x)$ is Lipschitz on $S_{T, n}$. Thus it is sufficient to prove the Lipschitz continuity of $u(t, x)$ in some neighborhood of hyperplane $\{t=0\}$. Define

$$
u_{i}(t, x)=\min _{\xi \in R^{n}}\left\{\varphi(\xi)+\frac{|x-\xi|^{2}}{4 a_{i} t}\right\}, i=1,2 .
$$

From (2.6) we have

$$
u_{1}(t, x) \leq u(t, x) \leq u_{2}(t, x) .
$$

Hopf's formula implies that $u_{i}(t, x)$ satisfies the equations

$$
u_{i t}+a_{i} u_{i x}^{2}=0
$$

and the initial condition (1.2). It is known [9] that the generalized solutions in the sense of definition 2.1 of this problem exist and are unique. Let

$$
\left|u_{i}\left(t_{1}, x_{1}\right)-u_{i}\left(t_{2}, x_{2}\right)\right| \leq L\left(\left|t_{1}-t_{2}\right|+\left|x_{1}-x_{2}\right|\right)
$$

for any $\left(t_{i}, x_{i}\right) \in \bar{S}_{T}$. Then (2.10) implies

$$
u_{1}\left(t, x_{1}\right)-\varphi\left(x_{2}\right) \leq u\left(t, x_{1}\right)-\varphi\left(x_{2}\right) \leq u_{2}\left(t, x_{1}\right)-\varphi\left(x_{2}\right) .
$$


Consequently

$$
\left|u\left(t, x_{1}\right)-\varphi\left(x_{2}\right)\right| \leq \max _{i=1,2}\left|u_{i}\left(t, x_{1}\right)-\varphi\left(x_{2}\right)\right| \leq L\left(|t|+\left|x_{1}-x_{2}\right|\right) .
$$

Thus $u(t, x)$ is generalized solution in the sense of definition 2.1. The theorem 3 [9] implies the unique of such solutions, so, any solution with the bounded and Lipschitz initial data (1.2) can be represented by formula (2.7). Now we prove that $u(t, x)$ corresponding to the bounded lower semicontinuous initial function $\varphi(x)$ is unique. The generalized solution of $(1.1),(1.2)$ in this case is defined below.

DeFinition 2.4. The bounded and Lipschitz continuous on $S_{T, n}$ function is called the generalized solution of $(1.1),(1.2)$ if $u(t, x)$ solves (1.1) a.e. on $S_{T}$ and satisfies:

$$
\Delta_{l}^{2} u(t, x)=u(t, x+l)-2 u(t, x)+u(t, x-l) \leq C_{n}|l|^{2},
$$

for any $(t, x),(t, x+l),(t, x-l) \in S_{T}^{n}$,

$$
\lim _{t \rightarrow 0} u(t, x)=\varphi(x)
$$

From the estimate (2.9) we obtain the existence of solution of (1.1), (1.2) in the sense of definition 2.4. Let $\nu(t, x)$ is any other solution. We will show that it can be represented by formula (2.7). Notice, that $\nu_{\alpha}(t, x)=v(t+\alpha, x)$, is the solution of (1.1), (1.2) with the bounded and Lipschitz initial data $\nu(\alpha, x)$. Thus

$$
\nu_{\alpha}(t, x)=\min _{\xi \in R^{n}}\left\{\nu(\alpha, \xi)+\frac{G^{2}(x, \xi)}{4 t}\right\} .
$$

Using (1.5), from the equation (1.1) we have that the sequence $\{\nu(\alpha, x)\}$ is increasing and according to lemma 3 [10] we can go to limit over minimum as $\alpha \rightarrow 0$. Thus $u=\nu$.

Suppose now that $\varphi(x)$ is unbounded and satisfying (1.6). Let $\sup _{|x \leq n|}|\varphi(x)| \leq$ $M_{n}$. Define

$$
\varphi_{n}(x)=\left\{\begin{array}{l}
\varphi(x),|x|<n, \\
-M_{n},|x| \geq n .
\end{array}\right.
$$

Let $u_{n}(t, x)$ be a solution of (1.1) defined by formula (2.7) corresponding to the initial function $\varphi_{n}(x)$. Then $\left|u_{n}(t, x)\right| \leq M_{n}$ and $u(t, x)=u_{n}(t, x)$ when $(t, x) \in K_{n}=\left\{x:|\xi| \leq n-2 \sqrt{2 a_{2} T M_{n}}\right\}$. From (1.6) we have that the balls $K_{n}$ cover up $R^{n}$. Now we will prove (2.1). According (1.6) we have that for any small $\mu>0$ there exists $c_{\mu}>0$ such that

$$
|\varphi(x)| \leq c_{\mu}+\mu|x|^{2} .
$$


Then (2.6) implies

$$
\begin{aligned}
|u(t, x)| & =\min _{\xi \in R^{n}}\left\{\phi(\xi)+\frac{G^{2}(x, \xi)}{4 t}\right\} \leq \min _{\xi \in R^{n}}\left\{c_{\mu}+\mu|\xi|^{2}+\frac{|x-\xi|^{2}}{4 a_{1} t}\right\} \\
& =c_{\mu}+\frac{\mu|x|^{2}}{4 \mu a_{1} t+1} .
\end{aligned}
$$

Consequently the function $u(t, x)$ is solution of (1.1), (1.2) in the sense of definition 2.2. The uniqueness of solution in this case immediate follows from the dependence of solution upon the bounded set of initial data.

\section{THE CALCULATION OF SOLUTIONS OF THE PROBLEMS (1.3), (1.2) AND (1.7), (1.2)}

Now we consider the equation (1.3) in $S=\left\{t \in(0,+\infty), x \in R^{n}\right\}$. Suppose $H(p) \in C^{2}(R)$,

$$
H^{\prime \prime}(p) \geq a>0
$$

and $\varphi(x)$ is Lipschitz on $R^{n}$. Let $u^{c}(t, x, \xi), c>0$, are the generalized solutions in the sense of definition 2.1 of (1.3) satisfying the initial condition

$$
u^{c}(0, x, \xi)=c|x-\xi| .
$$

From Hopf's formula follows that

$$
u^{c}(t, x, \xi)=\left\{\begin{array}{c}
-c(x-\xi)-t H^{\prime}(-c), x \leq \xi+t H^{\prime}(-c) \\
t \Phi\left(\frac{x-\xi}{t}\right), \xi+t H^{\prime}(-c) \leq x \leq \xi+t H^{\prime}(c) \\
c(x-\xi)-t H^{\prime}(c), x \leq \xi+t H^{\prime}(c)
\end{array}\right.
$$

Let $\left\{x_{k}\right\}=\{k h\}, h>0, k \in Z$. Define

$$
\begin{gathered}
\varphi_{h}^{c}(x)=\min _{k \in Z}\left\{\varphi\left(x_{k}\right)+c|x-\xi|\right\}, \\
u_{h}^{c}(t, x)=\min _{k \in Z}\left\{\varphi\left(x_{k}\right)+u^{c}\left(t, x, x_{k}\right)\right\} .
\end{gathered}
$$

Theorem 3.1. Let $H$ satisfies (2.9) and $\varphi(x)$ is uniformly Lipschitz on $R^{n}$ with the Lipschitz constant $L$. Then the functions $u_{h}^{c}(t, x), c \geq L$, are the generalized solutions of (1.3), (1.2) in $S$ with the initial function $\varphi_{h}^{c}(x)$ and

$$
\left|u_{h}^{c}(t, x)-u(t, x)\right| \leq c h,
$$

where $u(t, x)$ is a solution of (1.3), (1.2). 
Proof. In [5] we proved that the solution of Cauchy problem of (1.3), (1.2) can be represented by formula (3.3) when the initial function is uniformly Lipschitz on $R^{n}$. From Theorem 3 [9] we have

$$
\left|u_{h}^{c}(t, x)-u(t, x)\right| \leq \sup _{x \in R^{n}}\left|\varphi_{h}^{c}(x)-\varphi(x)\right| \leq c h .
$$

Remark 3.1. According to Theorem 1 [9] we have that minimum in (3.3) for any point $(t, x)$ can be searched in the finite set $\left[\frac{x-t H^{\prime}(-c)}{h}, \frac{x+t H^{\prime}(-c)}{h}\right] \cap Z$.

Remark 3.2. If the initial function $\varphi(x)$ is Lipschitz on $R^{n}$ and piecewise linear with the breaking points $\left\{x_{k}\right\}$ then the formula (3.3) gives the exact solution of (1.3), (1.2).

Now we give the formula for the solution $u(t, x)$ of conservation law (1.7) satisfying initial condition

$$
u(0, x)=\psi(x)=\left\{\begin{array}{l}
c_{i}, x \in\left[x_{i}, x_{i+1}\right), \\
c_{i+1}, x=x_{i+1}, i \in Z
\end{array}\right.
$$

where $\left|c_{i}\right| \leq c=$ const. Then the function $\varphi(x)=\int_{0}^{x} \psi(x) d x$ is Lipschitz continuous on $R^{n}$ and piecewise linear. According to Remark 3.2 we can give the solution of (1.3), (1.2) $w(t, x)$ by formula (3.3). Notice, that $u(t, x)=w_{x}(t, x)$ satisfies a.e. equation (1.7).

Definition 3.1. The $u(t, x)$ is a general solution of $(1.7),(1.2)$ if $\int_{0}^{x} u(t, x) d x$ is the general solution of (1.3), (1.2) in the sense of definition 2.1.

Now we will construct the solution of (1.7), (3.5). Define the function

$$
\varphi_{k}(x)= \begin{cases}c_{k}+c_{k-1}\left(x-x_{k}\right), & x \leq x_{k}, \\ c_{k}+c\left(x-x_{k}\right), & x \geq x_{k}\end{cases}
$$

when there are no breaking points to the left,

$$
\varphi_{k}(x)= \begin{cases}c_{k}-c\left(x-x_{k}\right), & x \leq x_{k} \\ c_{k}+c_{k}\left(x-x_{k}\right), & x \geq x_{k}\end{cases}
$$

when there are no breaking points to the right, and

$$
\varphi_{k}(x)= \begin{cases}c_{k}-c\left(x-x_{k}\right), & x \leq x_{k} \\ c_{k}+c_{k}\left(x-x_{k}\right), & x_{k} \leq x \leq x_{k+1} \\ c_{k}+c_{k}\left(x_{k+1}-x_{k}\right)+c\left(x-x_{k+1}\right), & x \geq x_{k+1}\end{cases}
$$


when the breaking point $x_{k}$ is between the other breaking points $x_{k-1}$ and $x_{k+1}$. Let $w_{k}(t, x)$ be the solution of (1.3), (1.2) corresponding the initial function $\varphi_{k}(x)$. We can calculate using $(3.3)$ that $w_{k}(t, x)$ is given by:

$$
\begin{aligned}
& w_{k}(t, x)=\left\{\begin{array}{c}
c_{k}+c_{k-1}\left(x-x_{k}\right)-t H^{\prime}\left(c_{k-1}\right), \\
x \leq x_{k}+t H^{\prime}\left(c_{k-1}\right), \\
c_{k}+t \Phi\left(\frac{x-x_{k}}{t}\right), \\
x_{k}+t H^{\prime}\left(c_{k-1}\right) \leq x \leq x_{k}+t H^{\prime}(c), \\
c_{k}+c\left(x-x_{k}\right)-t H^{\prime}(c), \\
x \geq x_{k}+t H^{\prime}(c),
\end{array}\right. \\
& w_{k}(t, x)=\left\{\begin{array}{c}
c_{k}+c\left(x-x_{k}\right)-t H^{\prime}(-c), \\
x \leq x_{k}+t H^{\prime}(-c), \\
c_{k}+t \Phi\left(\frac{x-x_{k}}{t}\right), \\
x_{k}+t H^{\prime}(-c) \leq x \leq x_{k}+t H^{\prime}\left(c_{k}\right), \\
c_{k}+c_{k}\left(x-x_{k}\right)-t H^{\prime}\left(c_{k}\right), \\
x \geq x_{k}+t H^{\prime}\left(c_{k}\right),
\end{array}\right. \\
& w_{k}(t, x)=\left\{\begin{array}{c}
c_{k}-c\left(x-x_{k}\right)-t H^{\prime}(-c), \\
x \leq x_{k}+t H^{\prime}(-c), \\
c_{k}+t \Phi\left(\frac{x-x_{k}}{t}\right), \\
x_{k}+t H^{\prime}(-c) \leq x \leq x_{k}+t H^{\prime}\left(c_{k}\right), \\
c_{k}+c_{k}\left(x-x_{k}\right)-t H^{\prime}\left(c_{k}\right), \\
x_{k}+t H^{\prime}\left(c_{k}\right) \leq x \leq x_{k+1}+t H^{\prime}\left(c_{k}\right), \\
c_{k}+c_{k}\left(x_{k+1}-x_{k}\right)+t \Phi\left(\frac{x-x_{k+1}}{t}\right), \\
x_{k+1}+t H^{\prime}\left(c_{k}\right) \leq x \leq x_{k+1}+t H^{\prime}(c), \\
c_{k}+c_{k}\left(x_{k+1}-x_{k}\right)+c\left(x-x_{k+1}\right)-t H^{\prime}(c), \\
x \geq x_{k+1}+t H^{\prime}(c) .
\end{array}\right.
\end{aligned}
$$

Theorem 3.2. Let $H$ satisfies (3.1). Then the solution of (1.7), (3.5) on $S$ can be represented by formula

$$
u(t, x)=\frac{\partial}{\partial x} \min _{k \in I(t, x)} w_{k}(t, x),
$$

where $I(t, x)=\left[\frac{x-t H^{\prime}(-c)}{h}, \frac{x+t H^{\prime}(c)}{h}\right] \cap Z,\left|c_{i}\right| \leq c$. 


\section{REFERENCES}

[1] M. Bardi, L.C. Evans. On Hopf's formulas for solutions of Hamilton-Jacobi equations. Non. Analysis, 8 (11), 1984, 1373 - 1381.

[2] M.G. Crandall, P.L. Lions. Some properties of viscosity solutions of Hamilton-Jacobi equations. Trans. Amer. Math Soc., 277, 1983, $1-42$.

[3] M.G. Crandall, H. Ishii, P.L. Lions. User's guide to viscosity solutions. Bulletin of the Amer. Math. Soc., 27 (1), 1992, $1-67$.

[4] A. Douglis. Solutions in the large for multi-dimensional nonlinear partial deferential equations of first order. Ann. Inst. Fourier, Grenoble, 15 (2), 1965, $1-35$.

[5] G. Gudynas. Generalized solutions of non-linear equations of first order with the bounded and lower semicontinous initial function. Preprint Vilnius, 1990, 1 - 35. (in Russian)

[6] 6. G. Gudynas. Representation formula for solutions of Eikonal type. Nonlinear Analysis: Modeling and control, 6 , 2001, $49-56$.

[7] E. Hopf. Generalized solutions of non-linear equations of 1 order. Journ. of Math. and Mech., 14 (6), 1965, 951 - 974.

[8] H. Ishii. Representation of solutions of of Hamilton-Jacobi equations. Non. Analysis:Modeling and Control, 12 (2), 1986, 121 - 146.

[9] Kruzkov S.N.. Generalized solutions of the multi-dimensional nonlinear partial differential equations of first order. I. Math. sbornik, 70 (112), 1966, $394-415$.

[10] Kruzkov S.N. Generalized solutions of the multi-dimensional nonlinear partial differential equations of first order. II Math. sbornik 72 (114), 1967, 108 - 134

\section{Apie Hamiltono-Jakobi sprendinių išraiškas tam tikroms pradinėms sąlygoms}

G. Gudynas

Nagrinėjami Koši uždaviniai lygtims

$$
\begin{aligned}
& u_{t}+\left(A(x) u_{x}, u_{x}\right)=0, \\
& u_{t}+H\left(u_{x}\right)=0 \\
& u_{t}+H(u)_{x}=0
\end{aligned}
$$

su pradine sąlyga

$$
u(0, x)=\varphi(x) .
$$

Iškilių hamiltonianų atveju yra užduotos formulès Koši uždavinių sprendiniams: pirmosios lygties atveju, kai $\varphi(x)$ yra pusiau tolydi iš apačios ir

$$
\lim _{|x| \rightarrow \infty}|x|^{-2}|\varphi(x)|=0
$$

antrosios lygties atveju pradine funkcija tolydi Lipšico prasme ir yra laužtė, trečiosios lygties atveju pradinè funkcija aprěžta ir dalimis pastovi. 